\title{
Secondary Structure Prediction of Chicken Egg White Ovomucoid
}

\author{
Tsukasa Matsuda, Kenji Watanabe and Yasushi Sato \\ Department of Food Science and Technology, Faculty of Agriculture, \\ Nagoya University, Nagoya 464, Japan \\ Received July 28,1980
}

\begin{abstract}
The secondary structure of chicken egg white ovomucoid (186 residues) was predicted from the amino acid sequence by the method of Chou and Fasman (P. Y. Chou and G. D. Fasman, Biochemistry, 13, 222 (1974)). The ovomucoid $\alpha$-helix regions were predicted by the method of Lewis et al. (P. N. Lewis et al., Proc. Natl. Acad. Sci. U.S., 65, 810 (1970)). The relative composition of the secondary structure obtained by the former method was: $\alpha$-helix ( 7 regions) $27 \%, \beta$-structure ( 10 regions) $33 \%, \beta$-turn ( 11 regions) $23 \%$ and random coil $17 \%$. In the predicted conformation, there were some $\alpha$-helical and $\beta$-structure segments that tended to be segregated along the polypeptide chain. These results indicate that the ovomucoid may be classified into the $\alpha+\beta$ type protein group and that the reactive site $\left(\operatorname{Arg}^{89} \unrhd \mathrm{Ala}^{90}\right.$ ) of trypsin inhibi tion is probably situated at the $\mathrm{C}$-terminal $\beta$-structure end. In comparisons of the predicted structures of the three domains in ovomucoid, domains I and II were suggested to be partially homologous to one another and not to domain III.
\end{abstract}

Chicken egg white ovomucoid is a glycoprotein with a trypsin inhibitory activity. ${ }^{11}$ The amino acid sequence of the ovomucoid has been determined by Kato et al., ${ }^{21}$ and it was demonstrated that the ovomucoid molecule consisted of three well separated domains, each being cross-linked by three disulfide bonds, and that amino acid sequences of the three domains were homologous to each other. ${ }^{2,3)}$ The reactive site of trypsin inhibition was also reported to be $\mathrm{Arg}^{89} \unrhd \mathrm{Ala}^{90}$ in domain II. ${ }^{4)}$ It is of interest to predict the secondary structure of the ovomucoid in the light of the reactive site and the sequence homology among the three domains. We have already reported the composition of the secondary structure of the ovomucoid and its three domains calculated from circular dichroism (CD) data, though the CD data provided only the percentage of each structural element. ${ }^{5)}$

During the last several years, a number of predictive methods for the secondary structure of proteins have been reported. ${ }^{6}$ In this paper, we present the ovomucoid secondary structure as predicted essentially according to the method of Chou and Fasman,7) then refined by the method of $\operatorname{Lim},{ }^{81}$ and the helix probability profile obtained by the method of Lewis et al. ${ }^{97}$ and Lewis and Scheraga, ${ }^{10}$ and discuss the conformational characteristics of the ovomucoid, the secondary structure at the reactive site and the structural homology among the three domains.

\section{METHODS}

The amino acid sequence of the ovomucoid used for conformational predictions, with the carbohydrateattachment sites, was as shown by Kato et al. ${ }^{2}$ and Kato $^{3)}$ (Fig. 1).

\section{Method of Chou and Fasman}

1) $\alpha$-Helix and $\beta$-structure regions. $\alpha$-Helix and $\beta$-structure regions were essentially determined by the predictive rules of Chou and Fasman. ${ }^{7)}$ For the conformational assignments of individual amino acids and the calculation of average conformational potentials, $\left\langle\mathrm{P}_{\alpha}\right\rangle$ and $\left\langle\mathrm{P}_{\beta}\right\rangle$, the data of Fasman et al. ${ }^{11)}$ were used. When there were overlapping predictions, the segments predicted in duplicate were determined to be in a helical or $\beta$-structure conformation according to their average conformational potentials. The minimum numbers of residues for $\alpha$-helix and $\beta$-structure predictions were usually set at six and five, respectively.

2) $\beta$-Turn regions. The relative probability, $P_{t}$, that a tetrapeptide forms a $\beta$-turn was given by Lewis et al. $^{12)}$

$$
P_{t}=f_{i} \cdot f_{i+1} \cdot f_{i+2} \cdot f_{i+3}
$$

In this expression, the $f \mathrm{~s}^{\prime}$ represent the frequency of 


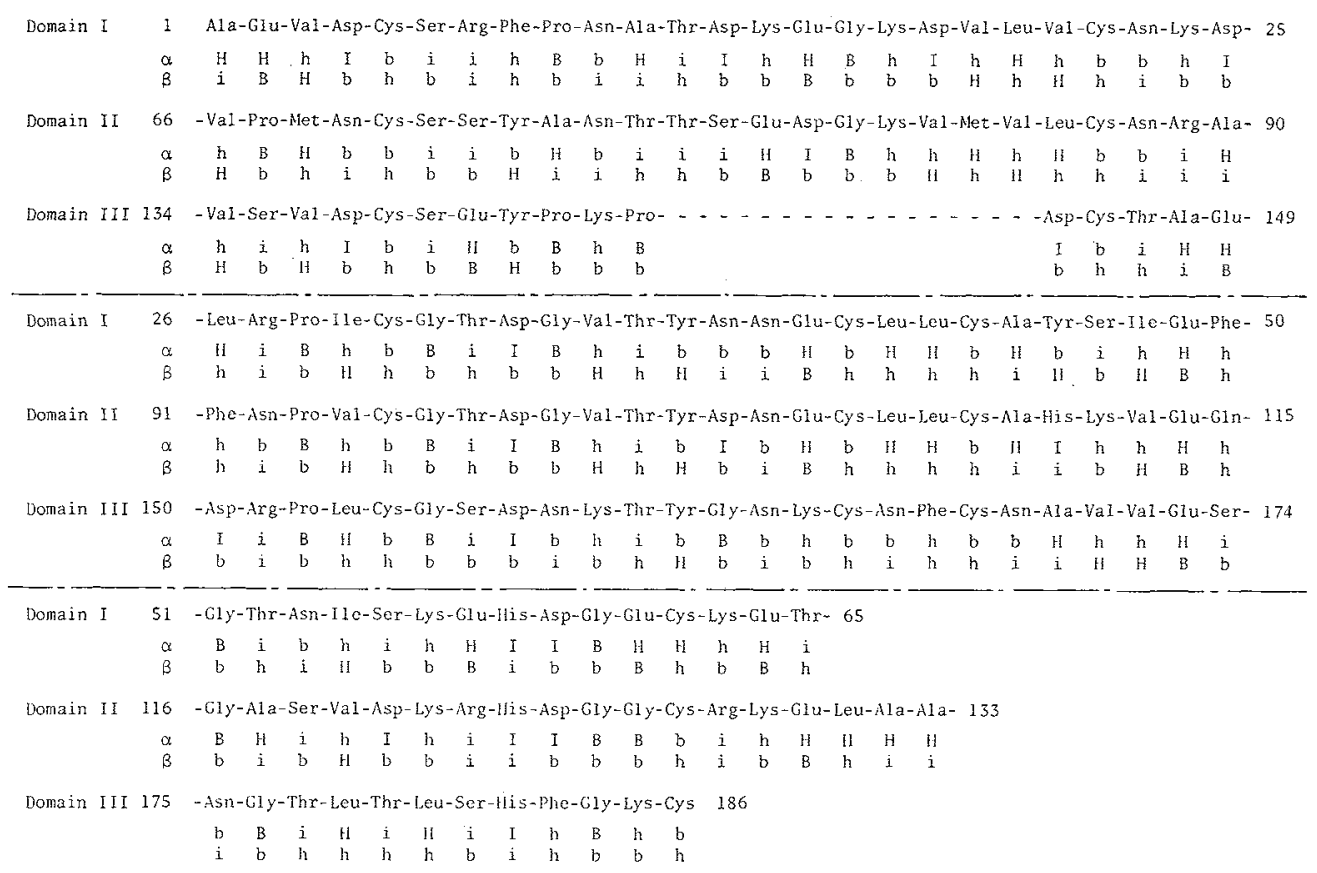

Frg. 1. Amino Acid Sequence of Chicken Ovomucoid and Assignments of Helical and $\beta$-Structure Potentials for Each Residue.

The amino acid sequence data were established by Kato et al..$^{2}$ and Kato. ${ }^{3)}$ To maximize the sequence homologies among the three domains, the sequence was separated and shifted according to the method of Kato et al. ${ }^{2)}$ Assignments in the first and second rows under each residue refer respectively to helical and $\beta$-structure potentials ( $H$, strong former, $h$, former, $I$, weak former, $i$, indifferent, $b$, breaker, $B$, strong breaker) as defined by Fasman et al. ${ }^{11)}$

occurrence for a certain residue at the first, second, third and fourth positions of a $\beta$-turn and were evaluated by Chou and Fasman. ${ }^{13}$ ) In the case of ovomucoid, the average value of $P_{t}$ is $1.165 \times 10^{-4}$. Therefore, according to the method of Chou and Fasman ${ }^{13}$ ) we took $P_{t}=2.33 \times 10^{-4}$ as the minimum value of the probability for a given tetrapeptide to adopt the $\beta$-turn conformation, and this value was regarded as the cutoff value in predicting a $\beta$-turn in the ovomucoid molecule.

Method of Lewis et al. The helix probability profile of the ovomucoid was obtained by the methods of Lewis et al. ${ }^{97}$ and Lewis and Scheraga. ${ }^{10)}$ Assignment of amino acid residues into three categories (former, independent and breaker) according to helix forming power is based on the method of Lewis and Scheraga. ${ }^{14)}$ The probabilities, $\mathrm{P}_{\mathrm{H}}(\mathrm{i}), \mathrm{P}_{\mathrm{ch}}(\mathrm{i})$ and $\mathrm{P}_{\mathrm{hc}}(\mathrm{i})$, were calculated using the values of $\sigma\left(5 \times 10^{-4}\right)$ and $\mathrm{s}$ (helix formers $s=1.05$, helix independents $s=1.00$ and helix breakers $s=0.385)$. $P_{F i}(i)$ is the probability that the $i$-th residue in a peptide chain is in a helix state. $P_{c h}(i)$ is the probability that the $i$-th residue is in a helix state and preceded by a residue in a coil state. $P_{\mathrm{be}}(\mathrm{i})$ is the probability that the $i$-th residue is in a coil state and preceded by a residue in a helix state.

\section{RESULTS AND DISCUSSION}

\section{Chou-Fasman prediction}

The predicted $\alpha$-helix and $\beta$-structure regions of the ovomucoid are summarized in Table I. There were 7 helical regions containing 51 residues and $10 \beta$-structure regions containing 62 residues. Region $144 \sim 151$ with a $\left\langle\mathbf{P}_{\alpha}\right\rangle$ value of 1.00 does not satisfy the condition that any segment predicted as a helical conformation must have a $\left\langle\mathbf{P}_{\alpha}\right\rangle$ value above 1.03. However, an $\alpha$-helix was predicted for this segment because Pro $^{144}$, Asp ${ }^{145}$ and $\mathrm{Arg}^{151}$ are good indicators of a helical formation; Pro and Asp prefer the N-terminal helical end and Arg prefers the C-terminal helical end, and in such cases an $I_{\alpha}$ assignment is given to these 
Table I. Conformational Prediction of Ovomucoid Computed for $\alpha$-Helix AND $\beta$-STRucture Regions: $\left\langle\mathrm{P}_{\alpha}\right\rangle$ AND $\left\langle\mathrm{P}_{\beta}\right\rangle$ VAlues

\begin{tabular}{|c|c|c|c|c|}
\hline & \multicolumn{4}{|c|}{ Ovomucoid (186 residues) } \\
\hline & Region* & Assignments & $\left\langle\mathrm{P}_{\alpha}\right\rangle^{\dagger}$ & $\left\langle\mathrm{P}_{\beta}\right\rangle^{+}$ \\
\hline \multirow[t]{7}{*}{$\alpha$-Helix } & $11 \sim 18(8)$ & $\mathrm{H}_{2} \mathrm{~h}_{2} \mathrm{I}_{2} \mathrm{iB}$ & 1.08 & 0.71 \\
\hline & $55 \sim 59(5)$ & $\mathrm{HhI}_{2} \mathrm{i}$ & 1.09 & 0.66 \\
\hline & $61 \sim 66(6)$ & $\mathrm{H}_{3} \mathrm{~h}_{2} \mathrm{i}$ & 1.13 & 0.93 \\
\hline & $110 \sim 115(6)$ & $\mathrm{H}_{2} \mathrm{~h}_{3} \mathrm{I}$ & 1.21 & 0.94 \\
\hline & $117 \sim 124(8)$ & $\mathrm{Hh}_{2} \mathrm{I}_{3} \mathrm{i}_{2}$ & 1.05 & 0.86 \\
\hline & $128 \sim 137(10)$ & $\mathrm{H}_{4} \mathrm{~h}_{3} \mathrm{~L}_{2}$ & 1.16 & 0.82 \\
\hline & $144 \sim 151(8)$ & $\mathrm{H}_{2} \mathrm{I}_{4} \mathrm{ib}$ & 1.00 & 0.77 \\
\hline \multirow[t]{10}{*}{$\beta$-Structure } & $19 \sim 23(5)$ & $\mathrm{H}_{2} \mathrm{~h}_{2} \mathrm{i}$ & 0.94 & 1.36 \\
\hline & $35 \sim 39(5)$ & $\mathrm{H}_{2} \mathrm{hi}_{2}$ & 0.78 & 1.23 \\
\hline & $41 \sim 48(8)$ & $\mathrm{H}_{2} \mathrm{~h}_{4} \mathrm{ib}$ & 0.97 & 1.20 \\
\hline & $50 \sim 54(5)$ & $\mathrm{Hh}_{2} \mathrm{ib}$ & 0.86 & 1.16 \\
\hline & $73 \sim 77(5)$ & $\mathrm{Hh}_{2} \mathrm{i}_{2}$ & 0.89 & 1.11 \\
\hline & $83 \sim 92(10)$ & $\mathrm{H}_{2} \mathrm{~h}_{4} \mathrm{i}_{4}$ & 1.06 & 1.19 \\
\hline & $100 \sim 104(5)$ & $\mathrm{H}_{2} \mathrm{hib}$ & 0.85 & 1.16 \\
\hline & $106 \sim 109(4)$ & $\mathrm{h}_{4}$ & 0.96 & 1.25 \\
\hline & $165 \sim 172(8)$ & $\mathrm{H}_{2} \mathrm{~h}_{3} \mathrm{i}_{3}$ & 0.93 & 1.22 \\
\hline & $177 \sim 183(7)$ & $h_{5} i b$ & 1.00 & 1.14 \\
\hline
\end{tabular}

$\alpha$-Helix: 7 regions, 51 residues, $27 \%$

$\beta$-Structure: 10 regions, 62 residues, $33 \%$

* The predicted $\alpha$-helix and $\beta$-structure regions are based on the rules of Chou and Fasman ${ }^{7}$ with conformational parameters updated for 29 proteins. ${ }^{11)}$

$+\left\langle\mathrm{P}_{\alpha}\right\rangle$ and $\left\langle\mathrm{P}_{\beta}\right\rangle$ are, respectively, the average conformational potential for the computed region to be in the helical and $\beta$-structure conformation.

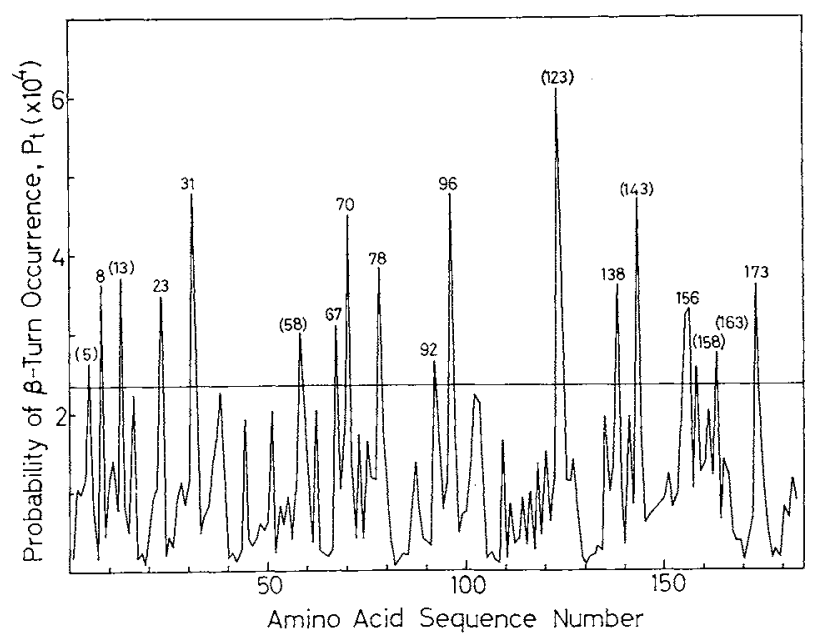

Fig. 2. Probability of Tetrapeptide $\beta$-Turns in Ovomucoid.

Of the peaks above the cut-off value $\left(2.33 \times 10^{-4}\right), 7$ were excluded (residue numbers shown in parentheses) as belonging to other predicted regions.

residues. ${ }^{7} \quad$ The $\left\langle\mathrm{P}_{\beta}\right\rangle$ value of 1.14 for region $177 \sim 183$ is higher than the $\left\langle\mathrm{P}_{\alpha}\right\rangle$ value of 1.00 . A $\beta$-structure is predicted for this region though $\alpha$-helicity can not be ruled out completely.
The relative probabilities that a tetrapeptide will form a $\beta$-turn were calculated with the equation described above and are depicted in the probability profile shown in Fig. 2. Eigh- 
Table II. Conformational Prediction of Ovomucoid Computed for $\beta$-Turn Regions

\begin{tabular}{|c|c|c|c|c|c|}
\hline Residues & Tetrapeptide* & $P_{t} \S$ & $\left\langle\mathbf{P}_{t}\right\rangle^{t}$ & $\left\langle\mathrm{P}_{\alpha}\right\rangle^{\dagger}$ & $\left\langle\mathrm{P}_{\beta}\right\rangle^{\dagger}$ \\
\hline $8 \sim 11$ & Phe-Pro-Asn-Ala & $3.64 \times 10^{-4}$ & 1.09 & 0.95 & 0.91 \\
\hline $23 \sim 26$ & Asn-Lys-Asp-Leu & $3.47 \times 10^{-4}$ & 1.12 & 1.01 & 0.87 \\
\hline $31 \sim 34$ & Gly-Thr-Asp-Gly & $4.78 \times 10^{-4}$ & 1.38 & 0.75 & 0.81 \\
\hline $67 \sim 70$ & Pro-Met-Asn-Cys & $3.09 \times 10^{-4}$ & 1.25 & 0.85 & 0.92 \\
\hline $70 \sim 73$ & Cys-Ser-Ser-Tyr & $4.51 \times 10^{-4}$ & 1.28 & 0.73 & 1.04 \\
\hline $78 \sim 81$ & Ser-Glu-Asp-Gly & $3.85 \times 10^{-4}$ & 1.29 & 0.97 & 0.60 \\
\hline $92 \sim 95$ & Asn-Pro-Val-Cys & $2.66 \times 10^{-4}$ & 1.22 & 0.75 & 1.08 \\
\hline $96 \sim 99$ & Gly-Thr-Asp-Gly & $3.63 \times 10^{-4}$ & 1.38 & 0.75 & 0.81 \\
\hline $138 \sim 141$ & Cys-Ser-Glu-Tyr & $3.63 \times 10^{-4}$ & 1.15 & 0.92 & 0.95 \\
\hline $156 \sim 159$ & Ser-Asp-Asn-Lys & $3.31 \times 10^{-4}$ & 1.31 & 0.90 & 0.73 \\
\hline $173 \sim 176$ & Glu-Ser-Asn-Gly & $3.64 \times 10^{-4}$ & 1.31 & 0.88 & 0.69 \\
\hline \multicolumn{6}{|c|}{$\beta$-Turns: 11 regions, 43 residues, $23 \%$} \\
\hline \multicolumn{6}{|c|}{$\begin{array}{l}\S P_{t} \text { is the probability of } \beta \text {-turn occurrence obtained by the method of Lewis et al }{ }^{12)} \text { with conformational } \\
\text { parameters update for } 29 \text { proteins. }{ }^{13)} \\
\left.+\mathrm{P}_{\mathrm{t}}\right\rangle,\left\langle\mathrm{P}_{\alpha}\right\rangle \text { and }\left\langle\mathrm{P}_{\beta}\right\rangle \text { are, respectively, the average conformational potential for the computed region to } \\
\text { be in the } \beta \text {-turn, helical and } \beta \text {-structure conformation. } \\
* \text { A } \beta \text {-turn is predicted for a tetrapeptide if the following conditions are met: }\left\langle\mathrm{P}_{\alpha}\right\rangle\left\langle\left\langle\mathrm{P}_{\mathrm{t}}\right\rangle ;\left\langle\mathrm{P}_{\beta}\right\rangle\left\langle\left\langle\mathrm{P}_{\mathrm{t}}\right\rangle \text {; }\right.\right.\end{array}$} \\
\hline
\end{tabular}

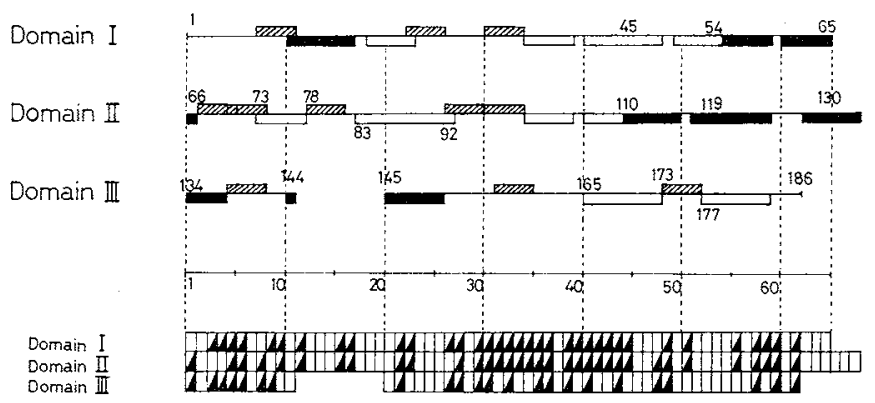

FIG. 3. Schematic Diagram of Predicted Secondary Structure of Ovomucoid.

Residues are represented in their respective conformational states: helical (畐), $\beta$-structure $(\square)$, $\beta$-turn ( 2$)$ and random coil (一). Identical amino acid residues in two or three domains ( $\square)$ are also shown below the diagram.

teen peaks were above the determined cut-off value of $2.33 \times 10^{-4}$. Of these peaks, 11 were predicted as $\beta$-turns, while the rest were excluded (residue numbers shown in parentheses in Fig. 2) as belonging to other predicted regions. The predicted $\beta$-turn regions of the ovomucoid are presented in Table II.

The predicted fraction of the ovomucoid secondary structure was as follows: $\alpha$-helix $27 \%, \beta$-structure $33 \%, \beta$-turn $23 \%$ and random coil $17 \%$. This composition was in relatively good agreement with that calculated from the $\mathrm{CD}$ data $(\alpha$-helix $26 \%, \beta$-structure $46 \%$, $\beta$-turn $10 \%$ and random coil $18 \%$ ), ${ }^{5}$ though there were some discrepancies. The Chou-
Fasman prediction is not perfect; it sometimes gives overlapping predictions for $\alpha$ helices and $\beta$-structures and there are some ambiguities in their rules. ${ }^{15)}$ The interpretation of the CD data in terms of a backbone conformation is not a straightforward matter either as described previously. ${ }^{5}$ ) Hence, some discrepancy will presumably be unavoidable with these methods.

A schematic diagram of the predicted secondary structure of ovomucoid is presented in Fig. 3. There were some $\alpha$-helix and $\beta$ structure segments that tended to be segregated along the polypeptide chain. According to this characteristic, it was predictable that the 
ovomucoid is a protein of the $\alpha+\beta$ type like lysozyme, papain, etc. ${ }^{16)}$ Each pair of two $\beta$ structure regions $(73 \sim 77$ and $83 \sim 92$, and $165 \sim 172$ and $177 \sim 183$ ) had a predicted $\beta$ turn between them, that is, $78 \sim 81$ and $173 \sim$ 176 , respectively, which might favor antiparallel $\beta$-structure formation in these regions.

Lim introduced the predictive approach based on the analysis of the hydrophobicity and size of the residue side chains, and the characterization of $\beta$-structure segments into "internal", "semi-surface" and "surface" $\beta$-structures. $^{8)}$ Based on this method an "internal" $\beta$-structure was predicted for region $82 \sim 89$, which consisted of five large hydrophobic, two large hydrophilic and one small hydrophilic residues. This result was in good accordance with that of the Chou-Fasman prediction $(\beta$ structure region $83 \sim 92$ ). Thus, it was sug-

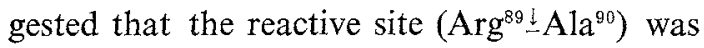
situated at the $\mathrm{C}$-terminal $\beta$-structure end.

Previously, Aubert et al. found that 37 carbohydrate peptide linkages including $O$ glycosidic $(N$-acetylgalactosamine $\rightarrow$ Ser $/$ Thr $)$ and $N$-glycosidic ( $N$-acetylglucosamine $\rightarrow$ Asn) bonds, characterized in 19 glycoproteins, were situated predominantly in $\beta$-turn regions. ${ }^{17}$ Beeley determined that four glycosylated Asn residues in the ovomucoid were very close to groups of amino acids that occurred with high frequency in $\beta$-turns. ${ }^{18)}$ Our finding of five glycosylated Asn residues in this work also indicated that tetrapeptides with high frequency in $\beta$-turns were closely related to all the sites of glycosylation except one $\left(\mathrm{Asn}^{53}\right)$, that is, three $\left(A s^{10}, A^{10} n^{69}\right.$ and $\left.A s n^{175}\right)$ of the five carbohydrate attachment sites were situated in $\beta$-turn regions and the other one $\left(\mathrm{Asn}^{75}\right)$ was very close to a $\beta$-turn region.

The predicted structures of the three domains in the ovomucoid are compared with each other in Fig. 3. Judging from our prediction, the three domains in the ovomucoid did not seem to be homologous to each other. The proportion of identical amino acid residues between domains I and II was $48 \%$. Moreover, in the light of the conformational potential of the secondary structure, the pro- portion of residues of identical conformational assignment (former; $H, h, I$, or indifferent; $i$, or breaker; $B, b$ ) reached $72 \%$ for $\alpha$-helix and $75 \%$ for $\beta$-structure regions. However, domains I and II were predicted to be only partially homologous in regard to the secondary structure. For example, for region $45 \sim$ 54 , the average conformational potentials were $\left\langle\mathrm{P}_{\alpha}\right\rangle=0.98$ and $\left\langle\mathrm{P}_{\beta}\right\rangle=1.08$, and the conformational assignments were $\left(\mathrm{H}_{2} \mathrm{~h}_{3} \mathrm{i}_{2} \mathrm{~b}_{2} \mathrm{~B}\right)_{\alpha}$ and $\left(\mathrm{H}_{3} \mathrm{~h}_{2} \mathrm{i}_{2} \mathrm{~b}_{2} \mathrm{~B}\right)_{\beta}$, whereas for region $110 \sim$ 119 , the average conformational potentials were $\left(\left\langle\mathrm{P}_{\alpha}\right\rangle=1.11\right.$ and $\left\langle\mathrm{P}_{\beta}\right\rangle=0.96$, and the conformational assignments were $\left(\mathrm{H}_{3} \mathrm{~h}_{4} \mathrm{IiB}\right)_{\alpha}$ and $\left(\mathrm{H}_{2} \mathrm{hi}_{3} \mathrm{~b}_{3} \mathrm{~B}\right)_{\beta}$. Thus, the predicted structures of region $45 \sim 54$ in domain $I$ and the corresponding region, $110 \sim 119$, in domain II were clearly different. The proportions of identical amino acid residues between domains I and III, and between domains II and III were no more than $38 \%$ and $32 \%$, respectively. In addition, the proportions of residues of identical conformational assignment were no more than $70 \%$ both between domains $I$ and III, and between domains II and III. These facts also suggested that the structural homology of domain III to the other domains might be inferior to that between domains I and II.

\section{Lewis et al. prediction.}

The helix probability profile of the ovomucoid obtained by the method of Lewis et al..$^{9,10}$ is shown in Fig. 4-A. The mean helix probability, $\theta_{\mathrm{H}}$, is represented by a horizontal line which intersects the ordinate. If the helix probability for the $i$-th residue, $\mathrm{P}_{\mathrm{H}}(i)$, equals or exceeds the mean helix probability, the residue is considered to be in the $\alpha$-helix conformation. Plots of the quantities $\mathrm{P}_{\mathrm{ch}}$ (i) and $P_{h c}(i)$ are shown in Fig. 4-B. The magnitude of peaks reflects the probability that a helix begins or ends at the $i$-th position. The prediction whether or not a particular residues is $\alpha$-helical was determined by, first, whether or not $\mathrm{P}_{\mathrm{H}}$ (i) exceeded $\theta_{\mathrm{H}}$ and, second, whether or not this residue lay in the regions defined by $\mathrm{P}_{\mathrm{ch}}$ (i) and $\mathrm{P}_{\mathrm{hc}}(\mathrm{i})$. The helical regions predicted in this manner are also shown as horizontal 


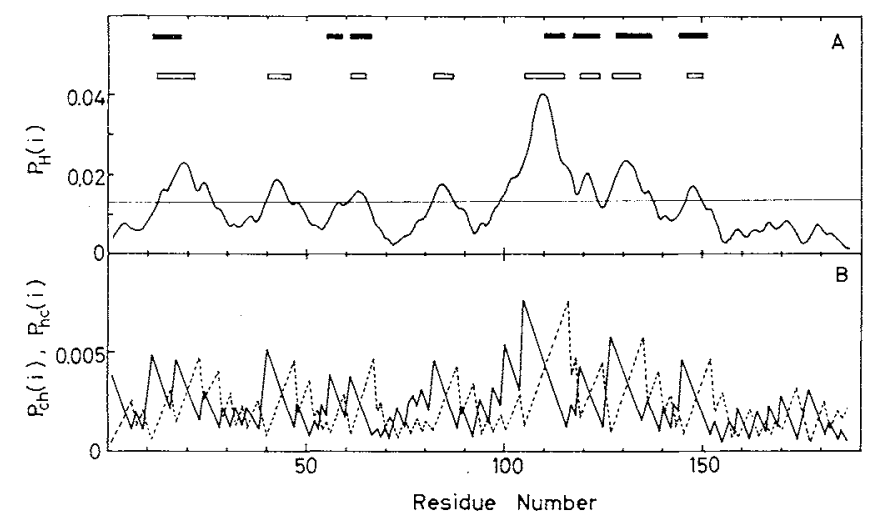

FIG. 4. Helix Probability Profile and Plots of $P_{\mathrm{ch}}(i)$ and $P_{\mathrm{hc}}(i)$ for Ovomucoid.

A : Helix probability profile. The horizontal line is the calculated values of the overall mean helix probability, $\theta_{\mathrm{F}}$, for the ovomucoid.

B: Plots of $P_{\mathrm{eh}}(i)(-)$ and $P_{\mathrm{hc}}(i)(-\cdots---)$.

The predicted helical regions, utilizing the $P_{\mathrm{H}}(i), P_{\mathrm{ch}}(i)$ and $P_{\mathrm{he}}(i)$ information, are shown as horizontal bars $(\square)$. The helical regions predicted by the method of Chou and Fasman ( $\square$ ) are also shown for comparison.

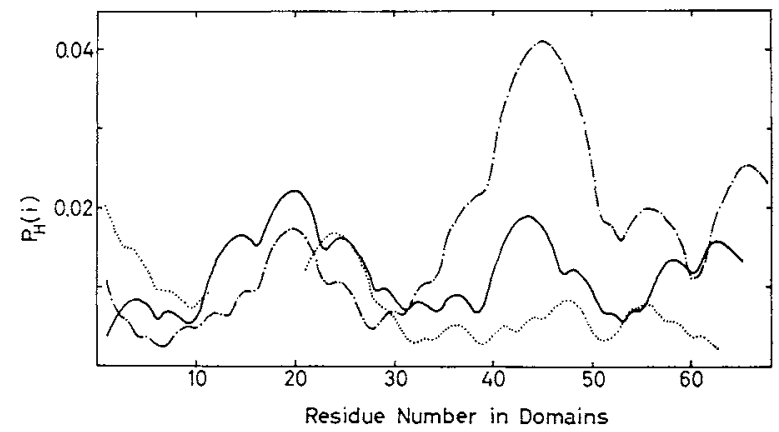

FIG. 5. Comparison of Helix Probability Profiles for Domains I (- - , II $(-\cdot-\cdot)$ and III (--) of Ovomucoid.

bars in Fig. 4-A. On comparison between the locations of helical regions determined by the Lewis et al. and Chou-Fasman methods as shown in Fig. 4, these two predictions were found to be in relatively good agreement.

Lenstra evaluated the secondary structure predictions for proteins obtained by the recently published methods (the joint histogram method of Argos et al., ${ }^{19)}$ the stereochemical method of $\mathrm{Lim}^{8}{ }^{8}$ the statistical mechanical method of Tanaka and Scheraga, ${ }^{15}$ and the statistical method of $\left.\mathrm{Nagano}^{20}\right)$ ) using the data of 33 proteins. $^{6}$. The comparison showed that the method of Tanaka and Scheraga, $\left.{ }^{15}\right)$ which was essentially the same as that of Lewis et $a l^{9,10)}$ for $\alpha$-helix prediction, yielded the least reliable results of the four. However, it should be noted that if the objects of prediction are limited to $\alpha$-helices in $\alpha+\beta$ proteins like insulin, papain, etc., the method of Tanaka and Scheraga is not less reliable than the others. ${ }^{6)} \quad$ The Chou-Fasman prediction and the $\mathrm{CD}$ analyses ${ }^{5}$ on the ovomucoid suggested that the ovomucoid was probably an $\alpha+\beta$ protein as described above. Hence, it seems reasonable to predict only $\alpha$-helix regions in the ovomucoid by the method of Lewis et al.

The helix probability profiles of the three domains in the ovomucoid are compared in Fig. 5. The profiles of domains I and II are similar to one another in their shape but do not necessarily correspond in their intensities. 
As seen in Fig. 5, on the N-terminal side the intensity in domain $I$ is stronger than in domain II, whereas on the C-terminal side the intensity in domain II is stronger than in domain I. The profile of domain III was evidently different from the others in both shape and intensity. Moreover, the helix probability profiles of domains I and II did not show the same extent of similarity as seen in the several species of Cytochrome C proteins, ${ }^{101}$ which were reported to be structurally homologous.

Consequently, the predicted secondary structure of the ovomucoid suggested that the ovomucoid molecule was an $\alpha+\beta$ protein and its domains I and II were only partially homologous to one another and not to its domain III.

\section{REFERENCES}

1) M. B. Rhodes, N. Bennet and R. E. Feeney, $J$. Biol. Chem., 235, 1686 (1960).

2) I. Kato, W. J. Kohr and M. Laskowski, Jr., "Regulatory Proteolytic Enzymes and Their Inhibitors," FEBS 11th Meeting, ed. by S. Magnusson, M. Ottesen, B. Faltmann, K. Danø and $\mathrm{H}$. Neurath, Pergamon Press, Oxford, New York, 1978, p. 197.

3) I. Kato, Protein, Nucleic Acid and Enzyme, 24, 667 (1979).
4) I. Kato, J. Schrode, K. A. Wilson and M. Laskowski, Jr., Protides Biol. Fluids, 23, 235 (1976).

5) K. Watanabe, T. Matsuda and Y. Sato, Biochim. Biophys. Acta, 667 (1981), in press.

6) J. A. Lenstra, Biochim. Biophys. Acta, 491, 333 (1977).

7) P. Y. Chou and G. D. Fasman, Biochemistry, 13, 222 (1974).

8) V. Lim, J. Mol. Biol., 88, 873 (1974).

9) P. N. Lewis, N. Gō, M. Gō, D. Kotelchuck and H. A. Scheraga, Proc. Nat. Acad. Sci. U.S.A., 65, $810(1970)$.

10) P. N. Lewis and H. A. Scheraga, Arch. Biochem. Biophys., 144, 576 (1971).

11) G. D. Fasman, P. Y. Chou and A. J. Adler, Biophys. J., 16, 1201 (1976).

12) P. N. Lewis, F. A. Momany and H. A. Scheraga, Biochim. Biophys. Acta, 303, 211 (1973).

13) P. Y. Chou and G. D. Fasman, J. Mol. Biol., 115, 135 (1977).

14) P. N. Lewis and H. A. Scheraga, Arch. Biochem, Biophys., 144, 584 (1971).

15) S. Tanaka and H. A. Scheraga, Macromolecules, 9, 168 (1976).

16) M. Levitt and C. Chothia, Nature, 261, 552 (1976).

17) J-P. Aubert, G. Biserte and M-H. LoucheuxLefebure, Arch. Biochem. Biophys., 175, 410 (1976).

18) J. G. Beeley, Biochem. J., 159, 335 (1976).

19) P. Argos, J. Schwarz and J. Schwarz, Biochim. Biophys. Acta, 439, 261 (1976).

20) K. Nagano, J. Mol. Biol., 109, 251 (1977). 